Tôhoku Math. Journ. 32 (1980), 411-417.

\title{
NOTES ON THE CANCELLATION OF RIEMANNIAN MANIFOLDS
}

\author{
HITOSHI TAKAGI
}

(Received July 10, 1979)

Introduction. Let $M, N$ and $B$ be Riemannian manifolds. Then, we have a question "Is $M$ isometric to $N$, if $M \times B$ is isometric to $N \times B$ ?" Uesu [1] proved that the answer is affirmative if $M$ and $N$ are complete and if $B$ is compact locally symmetric. In the present note, we shall show that the answer is affirmative also if the last condition on $B$ is replaced by one of the following (1) and (2):

(1) $B$ is simply connected and complete.

(2) $B$ is complete and $B$ has the irreducible restricted holonomy group.

The last assertion is stated as Theorems A and B in the next section.

We assume, in the present note, that all Riemannian manifolds are connected and $C^{\infty}$.

1. Proof of the theorems. First, we give some lemmas. Let $\Omega^{\prime}=$ $\{1, \cdots, r\}$ and $\Omega^{\prime \prime}=\{r+1, \cdots, n\}$. For a subset $\Omega \subset \Omega^{\prime} \cup \Omega^{\prime \prime}$, we denote by $S(\Omega)$ the symmetric group of $\Omega$. And, by $S_{n}$ and $S_{r}$, we denote $S\left(\Omega^{\prime} \cup \Omega^{\prime \prime}\right)$ and $S\left(\Omega^{\prime}\right)$, respectively. Let $G$ be a subgroup of $S_{r}$ and $\Omega_{0}^{\prime}=\left\{i \in \Omega^{\prime} \mid \tau(i)=i\right.$ for all $\tau \in G\}, \Omega_{1}^{\prime}=\left\{i \in \Omega^{\prime} \mid \tau(i) \neq i\right.$ for some $\left.\tau \in G\right\}$.

Lemma 1. Let $\sigma$ be an element of $S_{n}$.

(i ) If $\omega \in S\left(\sigma\left(\Omega_{0}^{\prime} \cup \Omega^{\prime \prime}\right)\right)$, then $(\omega \sigma) \tau(\omega \sigma)^{-1}=\sigma \tau \sigma^{-1}$ for all $\tau \in G$.

(ii) If $\sigma G \sigma^{-1} \subset S_{r}$, then $\sigma\left(\Omega_{1}^{\prime}\right) \subset \Omega^{\prime}$.

Proof. ( i ) We note $\tau\left(\Omega_{1}^{\prime}\right)=\Omega_{1}^{\prime}$ for all $\tau \in G$. If $i \in \Omega_{1}^{\prime}$, then $\omega \sigma(i)=$ $\sigma(i)$, and hence $\omega \sigma \tau \sigma^{-1} \omega^{-1}(\omega \sigma(i))=\omega \sigma(\tau(i))=\sigma \tau(i), \quad \sigma \tau \sigma^{-1}(\omega \sigma(i))=$ $\sigma \tau \sigma^{-1}(\sigma(i))=\sigma \tau(i)$. If $i \in \Omega_{0}^{\prime} \cup \Omega^{\prime \prime}$, then $\omega \sigma \tau \sigma^{-1} \omega^{-1}(\omega \sigma(i))=\omega \sigma \tau(i)=\omega \sigma(i)$ and $\left(\sigma \tau \sigma^{-1}\right)(\omega \sigma(i))=\omega \sigma(i)$, as $\omega \sigma(i)=\sigma(j)$ for some $j \in \Omega_{0}^{\prime} \cup \Omega^{\prime \prime}$. (ii) Assume $i \in \Omega_{1}^{\prime}$ and $\sigma(i) \in \Omega^{\prime \prime}$. Since $\sigma G \sigma^{-1} \subset S_{r}$, we have $\sigma \tau \sigma^{-1}(\sigma(i))=\sigma(i)$ and hence $\tau(i)=i$ for all $\tau \in G$, a contradiction.

q.e.d.

Throughout the present note, $I(M)$ denotes the group of all isometries of a simply connected and complete Riemannian manifold $M$.

Let $M_{1}=M_{2}=\cdots=M_{n}$ be a simply connected and complete Riemannian manifold whose homogeneous holonomy group is irreducible. Let $M$ be the direct product Riemannian manifold $M_{1} \times M_{2} \times \cdots \times M_{n}$. For 
each $\sigma \in S_{n}, \quad \lambda(\sigma): M \rightarrow M$ is defined by

$$
\lambda(\sigma)\left(x_{1}, \cdots, x_{n}\right)=\left(x_{\sigma^{-1}(1)}, \cdots, x_{\sigma^{-1}(n)}\right), \quad x_{i} \in M_{i} .
$$

Then, $\lambda(\sigma) \in I(M)$ and $\lambda: S_{n} \rightarrow I(M)$ is an isomorphism. Briefly we denote $\lambda(\sigma)$ by $\sigma$. Then $S_{n}$ is a subgroup of $I(M)$.

LEMMA 2. ( i ) $I(M)$ is generated by $S_{n}$ and $I\left(M_{1}\right) \times \cdots \times I\left(M_{n}\right)$. (ii) If $\sigma \in S_{n}$ and $\left(f_{1}, \cdots, f_{n}\right) \in I\left(M_{1}\right) \times \cdots \times I\left(M_{n}\right)$, then

$$
\sigma\left(f_{1}, \cdots, f_{n}\right) \sigma^{-1}=\left(f_{\sigma^{-1}(1)}, \cdots, f_{\sigma^{-1}(n)}\right),
$$

where $\left(f_{\sigma^{-1}(1)}, \cdots, f_{\sigma^{-1}(n)}\right) \in I\left(M_{1}\right) \times \cdots \times I\left(M_{n}\right)$. In particular, $I\left(M_{1}\right) \times \cdots \times$ $I\left(M_{n}\right)$ is a normal subgroup of $I(M)$.

Proof. ( $i$ ) is easily seen by the uniqueness of de Rham's decomposition (cf. Uesu [1] and Wolf [2]). (ii) $\sigma\left(f_{1}, \cdots, f_{n}\right) \sigma^{-1}\left(x_{1}, \cdots, x_{n}\right)=$ $\sigma\left(f_{1}, \cdots, f_{n}\right)\left(x_{\sigma(1)}, \cdots, x_{\sigma(n)}\right)=\sigma\left(f_{1}\left(x_{\sigma(1)}\right), \cdots, f_{n}\left(x_{\sigma(n)}\right)\right)=\left(f_{\sigma^{-1}(1)}\left(x_{1}\right), \cdots\right.$, $\left.f_{\sigma^{-1}(n)}\left(x_{n}\right)\right)=\left(f_{\sigma^{-1}(1)}, \cdots, f_{\sigma^{-1}(n)}\right)\left(x_{1}, \cdots, x_{n}\right)$.

q.e.d.

By the above lemma, $S_{n}$ is isomorphic to the quotient group $I(M) /$ $I\left(M_{1}\right) \times \cdots \times I\left(M_{n}\right)$. Let $\mu$ be the natural projection of $I(M)$ onto $I(M) /$ $I\left(M_{1}\right) \times \cdots \times I\left(M_{n}\right)$. Then, the image $\mu(\Gamma)$ of a subgroup $\Gamma$ of $I(M)$ is considered as a subgroup of $S_{n}$.

Let us decompose $M$ in Lemma 2 into $M^{\prime}$ and $M^{\prime \prime}$, where $M^{\prime}=$ $M_{1} \times \cdots \times M_{r}$ and $M^{\prime \prime}=M_{r+1} \times \cdots \times M_{n}$. Then $M=M^{\prime} \times M^{\prime \prime}$.

LEMMA 3. Let $\Gamma$ be a subgroup of $I\left(M^{\prime}\right)$ and $f \in I(M)$. If $f \Gamma f^{-1} \subset$ $I\left(M^{\prime}\right)$, then there exists $f^{\prime} \in I\left(M^{\prime}\right)$ satisfying $f^{\prime} h f^{\prime-1}=f h f^{-1}$ for all $h \in \Gamma$.

Proof. Let $G$ be the subgroup $\mu(\Gamma)$ which is a subgroup of $S_{r}$. Then, we may apply Lemma 1 with the other notations used. $f$ is written as $f=\sigma\left(f_{1}, \cdots, f_{n}\right)$ by Lemma 2 , where $\sigma \in S_{n}$ and $\left(f_{1}, \cdots, f_{n}\right) \in$ $I\left(M_{1}\right) \times \cdots \times I\left(M_{n}\right)$. Let $h$ be an element of $\Gamma$. Then $h$ is written as $h=\tau\left(h_{1}, \cdots, h_{r}, h_{r+1}, \cdots, h_{n}\right)$, where $\tau \in S_{r}, \quad\left(h_{1}, \cdots, h_{r}, h_{r+1}, \cdots, h_{n}\right) \in$ $I\left(M_{1}\right) \times \cdots \times I\left(M_{r}\right) \times I\left(M_{r+1}\right) \times \cdots \times I\left(M_{n}\right)$ and $h_{r+1}=\cdots=h_{n}=1$. Then we have

$$
f h f^{-1}=\sigma \tau \sigma^{-1}\left(f_{\tau \sigma^{-1}(1)} h_{\sigma^{-1}(1)} f_{\sigma^{-1}(1)}^{-1}, \cdots, f_{\tau \sigma^{-1}(n)} h_{\sigma^{-1}(n)} f_{\sigma^{-1}(n)}^{-1_{1}}\right),
$$

where $\left(f_{\tau \sigma^{-1}(1)} h_{\sigma^{-1}(1)} f_{\sigma^{-1} 1_{(1)}}^{-1}, \cdots, f_{\tau \sigma^{-1}(n)} h_{\sigma^{-1}(n)} f_{\sigma^{-1}(n)}^{-1_{1}}\right) \in I\left(M_{1}\right) \times \cdots \times I\left(M_{n}\right)$. Since $f h f^{-1} \in I\left(M^{\prime}\right)$, we have $\sigma \tau \sigma^{-1} \in S_{r}$ and $f_{\tau \sigma^{-1}(i)} h_{\sigma^{-1}(i)} f_{\sigma^{-1} 1_{(i)}}^{-1}=1$ if $i \in \Omega^{\prime \prime}$. On the other hand, by (ii) of Lemma 1 , if $i \in \Omega^{\prime \prime}$, then $\sigma^{-1}(i) \in \Omega_{0}^{\prime} \cup \Omega^{\prime \prime}$. Hence $\tau \sigma^{-1}(i)=\sigma^{-1}(i)$ and $h_{\sigma^{-1}(i)}=1$. Thus, if $j \in \sigma^{-1}\left(\Omega^{\prime \prime}\right) \cup \Omega^{\prime \prime}$, then $\tau(j)=j$ and $h_{j}=1$. We may assume for brevity that $\sigma^{-1}\left(\Omega^{\prime \prime}\right) \cap \Omega^{\prime}=$ $\{1, \cdots, s\}, s \leqq r$. Then $\Gamma \subset I\left(M_{s+1} \times \cdots \times M_{r}\right)$. 
Now, we define $\omega \in S\left(\left\{\sigma\left(j_{1}\right), \cdots, \sigma\left(j_{s}\right), \sigma(1), \cdots, \sigma(s)\right\}\right)$ by $\omega\left(\sigma\left(j_{k}\right)\right)=$ $\sigma(k)$ and $\omega(\sigma(k))=\sigma\left(j_{k}\right), k=1, \cdots, s$, where $\sigma^{-1}\left(\Omega^{\prime}\right) \cap \Omega^{\prime \prime}=\left\{j_{1}, \cdots, j_{s}\right\}$, $r+1 \leqq j_{1}<\cdots<j_{s} \leqq n$. And we define $f^{\prime} \in I\left(M^{\prime}\right)$ by $f^{\prime}=\omega \sigma(1, \cdots, 1$, $\left.f_{s+1}, \cdots, f_{r}, 1, \cdots, 1\right)$. Then, $f^{\prime}$ is the desired one. Indeed, as $\Gamma \subset$ $I\left(M_{s+1} \times \cdots \times M_{r}\right), \sigma\left(1, \cdots, 1, f_{s+1}, \cdots, f_{r}, 1, \cdots, 1\right) h\left(1, \cdots, 1, f_{s+1}, \cdots, f_{r}\right.$, $1, \cdots, 1)^{-1} \sigma^{-1}=f h f^{-1}$ for any $h \in \Gamma$. For any $h=\tau\left(h_{1}, \cdots, h_{n}\right) \in \Gamma$, $\sigma \tau \sigma^{-1} \in S(\{\sigma(s+1), \cdots, \sigma(r)\})$, as $\tau \in S(\{s+1, \cdots, r\})$. On the other hand, $f_{\tau \sigma^{-1}(j)} h_{\sigma^{-1}(j)} f_{\sigma^{-1}(j)}^{-1_{1}}=1$ for any $j \in\{\sigma(1), \cdots, \sigma(s), \sigma(r+1), \cdots, \sigma(n)\}$. Then, by $(*), \sigma\left(1, \cdots, 1, f_{s+1}, \cdots, f_{r}, 1, \cdots, 1\right) \Gamma\left(1, \cdots, 1, f_{s+1}, \cdots, f_{r}, 1\right.$, $\cdots, 1)^{-1} \sigma^{-1} \subset I\left(M_{\sigma(s+1)} \times \cdots \times M_{\sigma(r)}\right)$. But, as $\omega \in S\left(\left\{\sigma\left(j_{1}\right), \cdots, \sigma\left(j_{s}\right), \sigma(1)\right.\right.$, $\cdots, \sigma(s)\})$, we have $f^{\prime} h f^{\prime-1}=f h f^{-1}$.

Let $E^{n}$ be an $n$-dimensional Euclidean space. Let $E(n)=I\left(E^{n}\right)$. Then $E(n)$ is the semi-direct product group $O(n)+\boldsymbol{R}^{n}$, where $O(n)$ is the orthogonal group of the $n$-dimensional Euclidean vector space $\boldsymbol{R}^{n}$ and, if $(A, a),(B, b) \in E(n)$, the $(A, a)(B, b)=(A B, A b+a)$.

Lemma 4. Let $G$ be a subgroup of $O(n), A \in O(n)$ and $\bar{G}=A G A^{-1}$. Let $V=\left\{v \in \boldsymbol{R}^{n} \mid X v=v\right.$ for all $\left.X \in G\right\}$ and $W=\left\{w \in \boldsymbol{R}^{n} \mid Y w=w\right.$ for all $Y \in \bar{G}\}$. Then $A(V)=W$ and hence $A\left(V^{\perp}\right)=W^{\perp}$, where $V^{\perp}$ and $W^{\perp}$ are orthogonal complements in $\boldsymbol{R}^{n}$ of $V$ and $W$, respectively.

Let us consider $E^{n}$ as the direct product Riemannian manifold $E^{r} \times E^{n-r}$ of the Euclidean spaces $E^{r}$ and $E^{n-r}$.

LEMmA 5. Let $\Gamma$ be a subgroup of $E(r)=I\left(E^{r}\right)$ and $f \in E(n) . \quad$ If $f \Gamma f^{-1} \subset E(r)$, then there exists $f^{\prime} \in E(r)$ satisfying $f^{\prime} h f^{\prime-1}=f h f^{-1}$ for all $h \in \Gamma$.

Proof. Let $\bar{\Gamma}=f \Gamma f^{-1}, \quad V_{0}=\left\{v \in \boldsymbol{R}^{r} \mid \mu(h) v=v\right.$ for all $\left.h \in \Gamma\right\}$ and $W_{0}=\left\{w \in \boldsymbol{R}^{r} \mid \mu(\bar{h}) w=w\right.$ for all $\left.\bar{h} \in \bar{\Gamma}\right\}$, where $\mu$ is the projection $E(n) \rightarrow$ $O(n)$. Let $V=V_{0} \oplus \boldsymbol{R}^{n-r}$ and $W=W_{0} \oplus \boldsymbol{R}^{n-r}$. Then $\boldsymbol{R}^{r}=V^{\perp} \oplus V_{0}=$ $W^{\perp} \oplus W_{0}$ and $f$ is considered as a mapping $f: V^{\perp} \oplus V \rightarrow W^{\perp} \oplus W$, where $V^{\perp}$ and $W^{\perp}$ are orthogonal complements in $\boldsymbol{R}^{n}$ of $V$ and $W$, respectively. Then, by Lemma $4, \mu(f)\left(V^{\perp}\right)=W^{\perp}$ and $\mu(f)(V)=W$. Let $h=(X, x) \in \Gamma$ and $f=(A, a)$. Then $\left.X\right|_{V}=1$ and $x \in \boldsymbol{R}^{r}$. On the other hand, we-have (**) $f h f^{-1}=(A, a)(X, x)\left(A^{-1},-A^{-1} a\right)=\left(A X A^{-1},-A X A^{-1} a+A x+a\right)$.

Since $f h f^{-1} \in E(r)$, we have $A X A^{-1} \in O(r),-A X A^{-1} a+A x+a \in \boldsymbol{R}^{r}$ and $\left.A X A^{-1}\right|_{W}=1$. Here, $a$ is written as $a=a^{\prime}+a^{\prime \prime}$, where $a^{\prime} \in W^{\perp}$ and $a^{\prime \prime} \in W$. Then $-A X A^{-1} a+a=-A X A^{-1} a^{\prime}+a^{\prime} \in W^{\perp} \subset \boldsymbol{R}^{r}$. Thus $A x \in \boldsymbol{R}^{r}$, as $-A X A^{-1} a+A x+a \in \boldsymbol{R}^{r}$.

Now, let $U=\left\{v \in V_{0} \mid A v \in \boldsymbol{R}^{r}\right\}$. Then $V_{0}=U \oplus U^{\perp}$, where $U^{\perp}$ is the orthogonal complement of $U$ in $V_{0}$. Let $A^{\prime}$ be an element of $O(r)$ 
satisfying $\left.A^{\prime}\right|_{V^{\perp} \otimes U}=\left.A\right|_{V^{\perp} \otimes U},\left.A^{\prime}\right|_{R^{n-r}}=1$ and $A^{\prime}\left(U^{\perp}\right)=A(U)^{\perp}$, where $A(U)^{\perp}$ is the orthogonal complement of $A(U)$ in $W_{0}$. Then $f^{\prime}=\left(A^{\prime}, a^{\prime}\right) \in E(r)$ is the desired one. In fact, let $h=(X, x) \in \Gamma$. If $v \in V^{\perp}$, then $A X A^{-1}(A v)=A X(v)=A^{\prime} X A^{\prime-1}(A v) \quad$ as $\quad X v \in V^{\perp}$. If $v \in U$, then $A X A^{-1}(A v)=A v=A^{\prime} X A^{\prime-1}(A v)$ as $X v=v$. If $v \in R^{n-r}$, then $A X A^{-1}(A v)=$ $A v=A^{\prime} X A^{\prime-1}(A v)$ as $A v \in W, A^{\prime}\left(V_{0}\right)=W_{0}$ and $X v=v$. If $v \in U^{\perp}$, then $A X A^{-1}(A v)=A v=A^{\prime} X A^{\prime-1}(A v)$ as $X v=v$ and $A v \in R^{n-r}$. Then we have $A X A^{-1}=A^{\prime} X A^{\prime-1}$. Moreover, $A x=A^{\prime} x$ as $x \in V^{\perp} \oplus U$. Then $-A X A^{-1} a+$ $A x+a=-A^{\prime} X A^{\prime-1} a^{\prime}+A^{\prime} x+a^{\prime}$. Thus, by $(* *)$, we have $f^{\prime} h f^{\prime-1}=$ $f h f^{-1}$.

Lemma 6. Let $M, N$ and $B$ be complete Riemannian manifolds. If $M \times B$ is isometric to $N \times B$, then $\widetilde{M}$ is isometric to $\widetilde{N}$, where $\widetilde{M}$ and $\widetilde{N}$ are universal Riemannian covering manifolds of $M$ and $N$, respectively.

Proof. Let $p: \widetilde{M} \rightarrow M, p^{\prime}: \widetilde{N} \rightarrow N$ and $q: \widetilde{B} \rightarrow B$ be the universal Riemannian coverings. And let $\phi: M \times B \rightarrow N \times B$ be an isometry. Then, the covering $\phi \circ(p, q): \widetilde{M} \times \widetilde{B} \rightarrow N \times B$ has a lift $\tilde{\phi}: \widetilde{M} \times \widetilde{B} \rightarrow \widetilde{N} \times \widetilde{B}$, since $\widetilde{M} \times \widetilde{B}$ and $\widetilde{N} \times \widetilde{B}$ are simply connected. Then $\widetilde{\phi}$ is a covering and a local isometry as $\left(p^{\prime}, q\right) \circ \tilde{\phi}=\phi \circ(p, q)$. Hence, $\tilde{\phi}$ is an isometry. Thus $\tilde{M}$ is isometric to $\widetilde{N}$ by de Rham's decomposition theorem.

q.e.d.

Lemma 7. Let $\widetilde{M}$ be a simply connected and complete Riemannian manifold. Let $\Gamma$ and $\bar{\Gamma}$ be subgroups of $I(\widetilde{M})$ acting freely and properly discontinuously on $\widetilde{M}$. Then the quotient $\widetilde{M} / \Gamma$ is isometric to the quotient $\widetilde{M} / \bar{\Gamma}$ if and only if there exists an element $f \in I(\widetilde{M})$ satisfying $f \Gamma f^{-1}=\bar{\Gamma}$.

Proof. See Wolf [2].

REMARK. Let $\tilde{M}$ and $\tilde{N}$ be simply connected and complete Riemannian manifolds. Let $\tilde{\phi}: \widetilde{M} \rightarrow \widetilde{N}$ be an isometry. Let $\Gamma$ and $\bar{\Gamma}$ be subgroups of $I(\widetilde{M})$ acting freely and properly discontinuosly on $\widetilde{M}$. Then, $\Delta=\tilde{\phi} \Gamma \tilde{\phi}^{-1}$ and $\bar{\Delta}=\tilde{\phi} \bar{\Gamma} \tilde{\phi}^{-1}$ are subgroups of $I(\widetilde{N})$ acting freely and properly discontinuously on $\tilde{N}$. And $\tilde{\phi}$ induces natural isometries $\phi: \tilde{M} / \Gamma \rightarrow$ $\widetilde{N} / \Delta$ and $\bar{\phi}: \widetilde{M} / \vec{\Gamma} \rightarrow \widetilde{N} / \bar{\Delta}$. If there exists $f \in I(\widetilde{M})$ satisfying $\bar{\Gamma}=f \Gamma f^{-1}$, then $\bar{\Delta}=\left(\tilde{\phi} f \tilde{\phi}^{-1}\right) \Delta\left(\tilde{\phi} f \tilde{\phi}^{-1}\right)^{-1}$. Conversely, if there exists $g \in I(\tilde{N})$ satisfying $\bar{\Delta}=g \Delta g^{-1}$, then $\bar{\Gamma}=\left(\tilde{\phi}^{-1} g \tilde{\phi}\right) \Gamma\left(\tilde{\phi}^{-1} g \tilde{\phi}\right)^{-1}$. By Lemma $7, \widetilde{M} / \Gamma$ is isometric to $\widetilde{M} / \bar{\Gamma}$ if and only if $\tilde{N} / \Delta$ is isometric to $\tilde{N} / \bar{\Delta}$.

THEOREM A. Let $M$ and $N$ be complete Riemannian manifolds. Let $\widetilde{B}$ be a simply connected and complete Riemannian manifolds. If $M \times \widetilde{B}$ is isometric to $N \times \widetilde{B}$, then $M$ is isometric to $N$.

Proof. By Lemma 6, we may assume that $M$ and $N$ are isometric 
to $\tilde{M} / \Gamma$ and $\tilde{M} / \bar{\Gamma}$, respectively, where $\Gamma$ and $\bar{\Gamma}$ are subgroups of $I(\widetilde{M})$ acting freely and properly discontinuously on $\widetilde{M}$. Since $\widetilde{M} / \Gamma \times \widetilde{B}$ is isometric to $\widetilde{M} / \bar{\Gamma} \times \widetilde{B}$, there exists $f \in I(\widetilde{M} \times \widetilde{B})$ satisfying $f \Gamma f^{-1}=\bar{\Gamma}$ by Lemma 7. It is sufficient to prove that there exists $f^{\prime} \in I(\widetilde{M})$ satisfying $f^{\prime} h f^{\prime-1}=f h f^{-1}$ for all $h \in \Gamma$.

Now, by de Rham's decomposition theorem, we may assume that $\tilde{M}$ and $\widetilde{B}$ are isometric to the direct product Riemannian manifolds $N_{0} \times N_{1} \times \cdots \times N_{m} \times N^{*}$ and $B_{0} \times B_{1} \times \cdots \times B_{m} \times B^{*}$, respectively, which have the following properties (1) (4):

(1) $N_{0}, \cdots, N_{m}, N^{*}, B_{0}, \cdots, B_{m}$ and $B^{*}$ are all simply connected and complete.

(2) $N_{0} \times B_{0}$ is a Euclidean space.

(3) For each $i \in\{1, \cdots, m\}, \quad N_{i} \times B_{i}$ is a product of some Riemannian manifolds which are all isometric to one simply connected and complete Riemannian manifold $M_{i}$ whose homogeneous holonomy group is irreducible. And if $i \neq j$, then $M_{i}$ is not isometric to $M_{j}$.

(4) Any component of de Rham's decomposition of $N^{*} \times B^{*}$ has the irreducible homogeneous holonomy group. And any component of $N^{*}$ is not isometric to any of $B^{*}$.

By the above remark, we may suppose $\tilde{M}=N_{0} \times N_{1} \times \cdots \times N_{m} \times N^{*}$ and $\widetilde{B}=B_{0} \times B_{1} \times \cdots \times B_{m} \times B^{*}$. Moreover, we have a natural isometry $\phi: \widetilde{M} \times \widetilde{B} \rightarrow P=\left(N_{0} \times B_{0}\right) \times \cdots \times\left(N_{m} \times B_{m}\right) \times N^{*} \times B^{*}$. By the uniqueness of de Rham's decomposition, we have $I(P)=I\left(N_{0} \times B_{0}\right) \times \cdots \times I\left(N_{m} \times B_{m}\right) \times$ $I\left(N^{*}\right) \times I\left(B^{*}\right)$, (cf. Uesu [1]). Since $\Gamma$ and $\bar{\Gamma}$ are contained in $I(\widetilde{M})$, $\phi \Gamma \phi^{-1}$ and $\phi \bar{\Gamma} \phi^{-1}$ are contained in $I\left(N_{0}\right) \times \cdots \times I\left(N_{m}\right) \times I\left(N^{*}\right) \times\{1\}$, where $I\left(N_{i}\right)$ is interpreted as $I\left(N_{i}\right) \subset I\left(N_{i} \times B_{i}\right)$ for each $i \in\{0,1, \cdots, m\}$. Again, by the remark, we may consider $\phi \Gamma \phi^{-1}$ and $\phi \bar{\Gamma} \phi^{-1}$ as $\Gamma$ and $\bar{\Gamma}$, respectively. Then, it is sufficient to prove the following: Let $\Gamma$ and $\bar{\Gamma}$ be subgroups of $I\left(N_{0}\right) \times \cdots \times I\left(N_{m}\right) \times I\left(N^{*}\right) \times\{1\}$. If there exists $f \in I(P)$ satisfying $f \Gamma f^{-1}=\bar{\Gamma}$, then there exists $f^{\prime} \in I\left(N_{0}\right) \times \cdots \times I\left(N_{m}\right) \times I\left(N^{*}\right) \times$ \{1\} satisfying $f^{\prime} h f^{\prime-1}=f h f^{-1}$ for all $h \in \Gamma$.

Indeed, $f$ is written as $f=\left(g_{0}, g_{1}, \cdots, g_{m}, g^{*}, g^{* *}\right)$, where $g_{i} \in$ $I\left(N_{i} \times B_{i}\right), \quad g^{*} \in I\left(N^{*}\right)$ and $g^{* *} \in I\left(B^{*}\right) . \quad h$ is written as $h=\left(k_{0}, k_{1}, \cdots\right.$, $\left.k_{m}, k^{*}, 1\right)$, where $k_{i} \in I\left(N_{i}\right)$ and $k^{*} \in I\left(N^{*}\right)$. Then $f h f^{-1}=\left(g_{0} k_{0} g_{0}^{-1}, g_{1} k_{1} g_{1}^{-1}\right.$, $\left.\cdots, g_{m} k_{m} g_{m}^{-1}, g^{*} k^{*} g^{*-1}, 1\right)$. Now, the assertion is clear by Lemmas 3 and 5 .

q.e.d.

Theorem B. Let $B$ be a complete Riemannian manifold whose restricted homogeneous holonomy group is irreducible. And let $M$ and $N$ be complete Riemannian manifolds. If $M \times B$ is isometric to $N \times B$, then $M$ is isometric to $N$. 
Proof. Let $\widetilde{B}$ and $\widetilde{M}$ be universal Riemannian covering manifolds of $B$ and $M$, respectively. Then, by Lemma $6, M, N$ and $B$ are isometric to the quotients $\widetilde{M} / \Gamma, \widetilde{M} / \bar{\Gamma}$ and $\widetilde{B} / \Delta$, respectively. Then, by Lemma 7 , it is sufficient to prove: If there exists $f \in I(\widetilde{M} \times \widetilde{B})$ satisfying $f(\Gamma \times \Delta) f^{-1}=\bar{\Gamma} \times \Delta$, then there exists $f^{\prime} \in I(\widetilde{M})$ satisfying $f^{\prime} \Gamma f^{\prime-1}=\bar{\Gamma}$.

Let $M_{1} \times \cdots \times M_{n-1} \times M_{n}$ be de Rham's decomposition of $\tilde{M} \times \widetilde{B}$, where $\widetilde{M}=M_{1} \times \cdots \times M_{n-1}$ and $\widetilde{B}=M_{n}$. Then, by the uniqueness of de Rham's decomposition, $I(\widetilde{M} \times \widetilde{B})$ is generated by $I\left(M_{1}\right), \cdots, I\left(M_{n}\right)$ and by all permutations of $M_{i}$ 's which are isometric to each other, where we identify $M_{i}$ with $M_{j}$ by an isometry if $M_{i}$ is isometric to $M_{j}$ (cf. Uesu [1]). Moreover, we have a statement similar to (ii) of Lemma 2. Then $f$ is written as $f=\sigma\left(f_{1}, \cdots, f_{n-1}, f_{n}\right)$, where $\sigma \in S_{n}$ and $\left(f_{1}, \cdots, f_{n-1}, f_{n}\right) \in$ $I\left(M_{1}\right) \times \cdots \times I\left(M_{n-1}\right) \times I\left(M_{n}\right)$. Let $r=\sigma(n)$ and $s=\sigma^{-1}(n)$. Suppose $r \leqq n-1$ and hence $s \leqq n-1$. Then $M_{r}, M_{\mathrm{s}}$ and $M_{n}$ are isometric to each other. We shall prove

$$
\bar{\Gamma}=g_{r} \Delta_{r} g_{r}^{-1} \times\left(f \Gamma f^{-1} \cap \bar{\Gamma}\right) \quad \text { (the direct product group), }
$$

where $g_{r}=\left(1, \cdots, f_{n}, 1, \cdots, 1,1\right) \in I\left(M_{r}\right), \quad f \Gamma f^{-1} \cap \bar{\Gamma} \subset I\left(M_{1} \times \cdots \times M_{r-1} \times\right.$ $\left.M_{r+1} \times \cdots \times M_{n-1}\right)$ and $\Delta_{r}$ is the group $\Delta$ considered as a subgroup of $I\left(M_{r}\right)$. Let $h=\tau\left(h_{1}, \cdots, h_{n-1}, h_{n}\right) \in \Gamma \times \Delta$, where $\tau \in S_{n-1}$ and $\left(h_{1}, \cdots, h_{n-1}\right.$, $\left.h_{n}\right) \in I\left(M_{1}\right) \times \cdots \times I\left(M_{n-1}\right) \times I\left(M_{n}\right)$. Then

$$
f h f^{-1}=\sigma \tau \sigma^{-1}\left(f_{\tau \sigma^{-1(1)}} h_{\sigma^{-1}(1)} f_{\sigma^{-1}(1)}^{-1}, \cdots, f_{\tau \sigma^{-1}(n)} h_{\sigma^{-1}(n)} f_{\sigma^{-1}(n)}^{-1}\right),
$$

where $\left(f_{\tau \sigma^{-1}(1)} h_{\sigma^{-1}(1)} f_{\sigma^{-1}(1)}^{-1}, \cdots, f_{\tau \sigma^{-1}(n)} h_{\sigma^{-1}(n)} f_{\sigma^{-1}(n)}^{-1_{1}}\right) \in I\left(M_{1}\right) \times \cdots \times I\left(M_{n}\right)$. Since $\sigma \tau \sigma^{-1}(r)=r$, we have $\bar{\Gamma} \subset I\left(M_{r}\right) \times I\left(M_{1} \times \cdots \times M_{r-1} \times M_{r+1} \times \cdots \times M_{n-1}\right)$. Moreover $g_{r} \Delta_{r} g_{r}^{-1}=f \Delta f^{-1} \subset \bar{\Gamma} \cap I\left(M_{r}\right)$. Next, let $\bar{h} \in \bar{\Gamma}$. Then $\bar{h}$ is written as $\bar{h}=f h f^{-1}$, where $h \in \Gamma \times \Delta$. But $h$ is written as $h=h^{\prime} h^{\prime \prime}$, where $h^{\prime} \in \Gamma, h^{\prime \prime} \in \Delta$. Then $f h f^{-1}=f h^{\prime} f^{-1} f h^{\prime \prime} f^{-1}$. Since $f h^{\prime \prime} f^{-1} \in I\left(M_{r}\right) \cap \bar{\Gamma}$, we have $f h^{\prime} f^{-1} \in \bar{\Gamma}$. Hence $f h^{\prime} f^{-1} \in f \Gamma f^{-1} \cap \bar{\Gamma}$. By the above argument, it is evident that $f h^{\prime} f^{-1} \in I\left(M_{1} \times \cdots \times M_{r-1} \times M_{r+1} \times \cdots \times M_{n-1}\right)$.

Now, let $\omega$ be the transposition $(r, n) \in I\left(M_{r} \times M_{n}\right)$ and $f^{\prime}=g_{r} \omega \sigma\left(f_{1}, \cdots\right.$ $\left.f_{n-1}, 1\right) \in I(\widetilde{M})$. Then $f^{\prime}$ is the desired one. In fact, let $h=\tau\left(h_{1}, \cdots\right.$, $\left.h_{n-1}, 1\right) \in \Gamma$. As $: \tau \in S_{n-1}$, we have $f h f^{-1}=\sigma\left(f_{1}, \cdots, f_{n-1}, 1\right) h\left(f_{1}, \cdots\right.$, $\left.f_{n-1}, 1\right)^{-1} \sigma^{-1}$. On the other hand, as $f h f^{-1} \in \bar{\Gamma} \times \Delta$, we have $\sigma \tau \sigma^{-1}(n)=n$, that is, $\tau(s)=s$ and hence $f h f^{-1}$ is written as $f h f^{-1}=\left(\bar{h}^{\prime}, f_{s} h_{s} f_{s}^{-1}\right)$. where $\left(\bar{h}^{\prime}, 1\right) \in f \Gamma f^{-1} \cap \bar{\Gamma} \subset I\left(M_{1} \times \cdots \times M_{r-1} \times M_{r+1} \times \cdots \times M_{n-1}\right)$ and $(1, \cdots$, $\left.1, f_{s} h_{s} f_{s}^{-1}\right) \in \Delta \subset I\left(M_{n}\right)$. Then we have $\omega \sigma\left(f_{1}, \cdots, f_{n-1}, 1\right) h\left(f_{1}, \cdots, f_{n-1}\right.$, $1)^{-1} \sigma^{-1} \omega^{-1}=\left(1, \cdots, 1, f_{s} h_{s} f_{s}^{-1}, 1, \cdots, 1,1\right)\left(\bar{h}^{\prime}, 1\right) \in I\left(M_{r}\right) \times\left(f \Gamma f^{-1} \cap \bar{\Gamma}\right)$. Hence $f^{\prime} h f^{\prime-1}=g_{r}\left(1, \cdots, 1, f_{s} h_{s} f_{s}^{-1}, 1, \cdots, 1,1\right) g_{r}^{-1}\left(\bar{h}^{\prime}, 1\right) \in\left(g_{r} \Delta_{r} g_{r}^{-1}\right) \times\left(f \Gamma f^{-1} \cap \bar{\Gamma}\right)=$ $\bar{\Gamma}$. Thus, $f^{\prime} \Gamma f^{\prime-1} \subset \bar{\Gamma}$. By the above argument, it is evident that $f^{\prime} \Gamma f^{\prime-1}=\bar{\Gamma}$. 


\section{REFERENCES}

[1] K. UESU, On cancellation of compact Riemannian manifolds, to appear.

[2] J. A. Wolf, Spaces of Constant Curvature, McGraw-Hill, New York, 1967.

Department of Mathematics

College of General Education

TÔHOKU UNIVERSITY

SENDAI, 980 JAPAN 
